\title{
Density Bounds for the Sum of Divisors Function
}

\author{
By Charles R. Wall*, Phillip L. Crews and Donald B. Johnson
}

Abstract. Upper and lower bounds are presented for the density of the integers $n$ for which $\sigma(n) / n \geqq x$, where $\sigma(n)$ is the sum of divisors of $n$, and $1 \leqq x \leqq 5$.

1. Introduction. Let $\sigma$ be the sum of divisors function, and let $\varphi$ be Euler's function.

If $S$ is a subset of the positive integers, we define $S(N)$ to be the number of elements of $S$ among the first $N$ positive integers. The density $\delta S$ of $S$ is defined by

$$
\delta S=\lim _{N \rightarrow \infty} S(N) / N,
$$

provided the limit exists.

In this paper, we present upper and lower bounds for the density function

$$
A(x)=\delta\{n: \sigma(n) / n \geqq x\}
$$

of a real variable $x$. Davenport [2] proved that $A(x)$ exists, and is continuous, for all real $x$. Our technique is a refinement of that used by Behrend [1] to bound $A(2)$, the density of the abundant numbers. Our calculations were performed on an IBM 360/40 computer, and required 26 minutes of machine time.

2. Techniques. For $j$ and $k$ positive integers, we define

$$
A(x, j, k)=\delta\{n: j \mid n,(n / j, k)=1 \text { and } \sigma(n) / n \geqq x\} .
$$

The existence of the functions $A(x, j, k)$ may be proved by a slight modification of Davenport's proof [2] of the existence of

$$
A(x)=A(x, 1,1) .
$$

We remark that we may, without loss of generality, take $k$ to be squarefree here. It is not difficult to show that if $k$ is a fixed squarefree integer, then

$$
A(x)=\sum A(x, j, k),
$$

where the summation is over all $j$ composed of the primes which divide $k$. The same proof shows that if $k$ divides $k^{\prime}$, then

$$
A(x, 1, k)=\sum A\left(x, j, k^{\prime}\right),
$$

where the summation is over all $j$ composed of primes dividing $k^{\prime}$ but not $k$.

Received April 15, 1971.

AMS 1970 subject classifications. Primary 10H25, 10K20; Secondary 10A20.

Key words and phrases. Density, sum of divisors, arithmetic functions.

* Research partially supported by NSF Grant GP-7129.

Copyright $\odot 1972$, American Mathematical Society 
Assume henceforth that $k$ is squarefree and that any prime divisor of $j$ also divides $k$. Behrend [1] showed that if $\sigma(j) / j \geqq x$, then

$$
A(x, j, k)=\varphi(k) / j k \quad(x \leqq \sigma(j) / j)
$$

and if $\sigma(j) / j<x$, then

$$
A(x, j, k) \leqq \frac{\sigma(j) / j}{x-\sigma(j) / j} \cdot \frac{M}{j} \quad(x>\sigma(j) / j)
$$

where

$$
M=\frac{\varphi(k)}{k}\left\{-1+\frac{\pi^{2}}{6} \prod_{p \mid k}\left(1-p^{-2}\right)\right\} .
$$

We also have the trivial bound $A(x, j, k) \leqq \varphi(k) / j k$, which is better than (4) if $x-$ $\sigma(j) / j$ is small and positive.

Dedekind's function $\psi$ is defined by

$$
\psi(n)=n \prod_{p \mid n}(1+1 / p)
$$

this function is clearly multiplicative, whence

$$
\psi(n) \leqq \sigma(n),
$$

with equality if and only if $n$ is squarefree. It can be shown with little difficulty that

$$
2 \psi(n) \geqq n+\sigma(n) .
$$

Wall [3] has investigated the functions

$$
B(x, j, k)=\delta\{n: j \mid n,(n / j, k)=1 \text { and } \psi(n) / n \geqq x\},
$$

all of which exist and are continuous, and $B(x)=B(x, 1,1)$. It is clear from $(5)$ and (6) that

$$
B(x, j, k) \leqq A(x, j, k) \leqq B((x+1) / 2, j, k)
$$

for all $x, j$ and $k$. The relationship (7) is significant because the functions $B(x, j, k)$ are easier to bound than are the $A(x, j, k)$.

We set $k=2 \cdot 3 \cdot 5 \cdot 7 \cdot 11 \cdot 13 \cdot 17$ and let $j$ be composed of the primes $7,11,13$ and 17 with the exponent on each prime dividing $j$ being 0 or 1 or at least 2 . Using (2), (3) and (4), we obtained upper and lower bounds for $A(x, 1,30)$. We sharpened these bounds by use of (7) whenever possible.

Suppose that every prime which divides $j$ also divides $k$, and define $y=x j / \sigma(j)$. It is not difficult to show that

$$
A(x, j, k)=j^{-1} A(y, 1, k) .
$$

Using our bounds for $A(x, 1,30)$ with (1) and (8) and $k=30$, we obtained our bounds for $A(x)$ by summing over $j=2^{a} 3^{b} 5^{c}$, subject to $0 \leqq a \leqq 6$ or $a \geqq 7,0 \leqq b \leqq 3$ or $b \geqq 4$, and $0 \leqq c \leqq 2$ or $c \geqq 3$.

3. Results. Our bounds for $x=1(.1) 5$ are given in Table I and are illustrated by Fig. 1. Bounds for $x=1(.01) 5$ have been placed in the UMT file. 


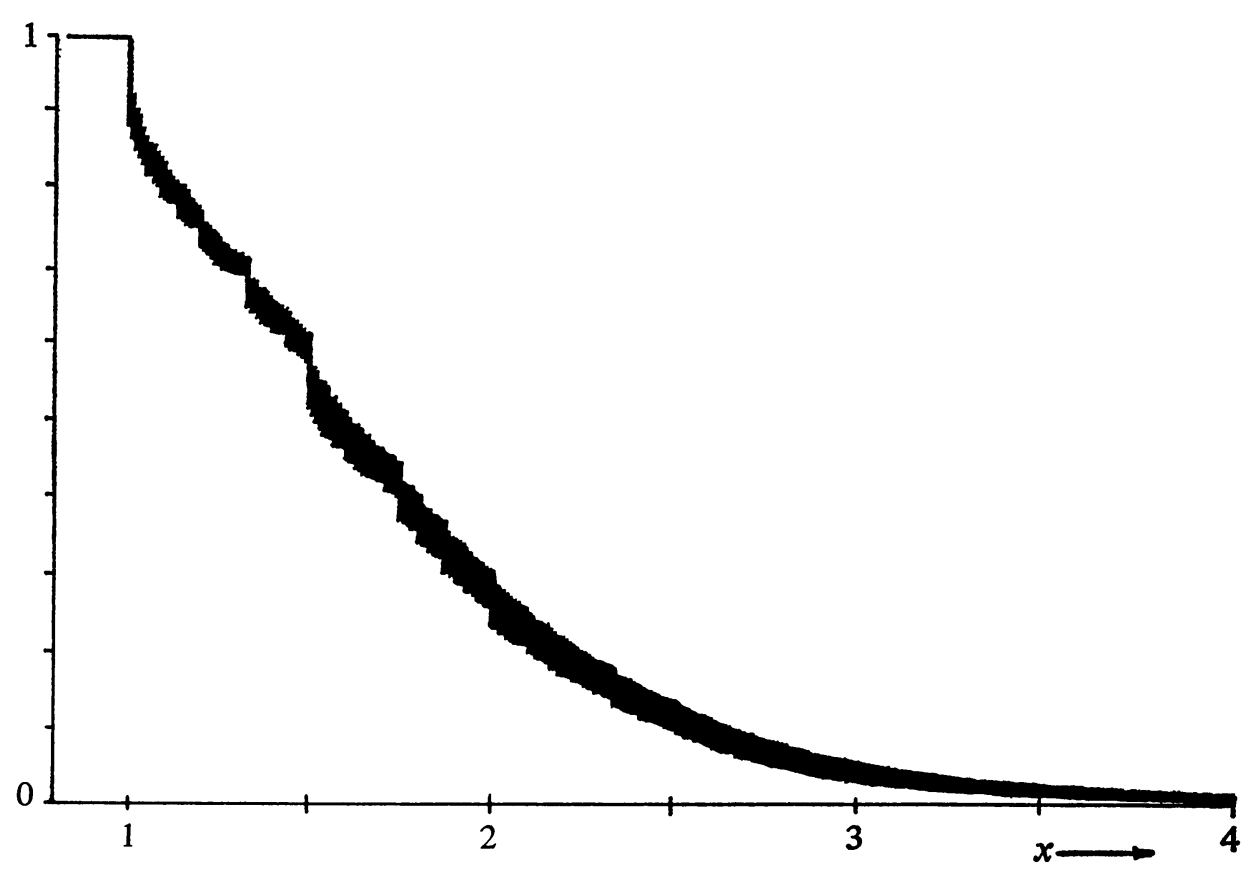

Figure 1. $A(x)$ lies in the shaded area

Because the density of the abundant numbers is of particular interest, we improved our lower bound for $A(2)$ to 0.2441 by observing that $\sigma(n) / n \geqq 2$ if $n$ is a multiple of any of the following integers: $2 \cdot 3,2^{2} 5,2 \cdot 5 \cdot 7,2 \cdot 5 \cdot 11 p(13 \leqq p \leqq 41), 2 \cdot 5 \cdot 13 p$ $(17 \leqq p \leqq 31), 2 \cdot 5 \cdot 17 \cdot 19,2^{2} 7,2 \cdot 7 \cdot 11 \cdot 13,2^{3} 11,2^{2} 11 \cdot 13,2^{2} 11 \cdot 17,2^{2} 11 \cdot 19,2^{4} p$ $(13 \leqq p \leqq 31), 2^{3} 13 p(17 \leqq p \leqq 41), 2^{3} 17 p(19 \leqq p \leqq 41), 2^{3} 19 p(23 \leqq p \leqq 41)$, $2^{3} 23 p(29 \leqq p \leqq 41), 2^{3} 29 \cdot 31,2^{5} 37,2^{5} 41,2 \cdot 5^{2} 11,2 \cdot 5^{2} 13,3^{2} 5 \cdot 7,3 \cdot 5^{2} 7 p(11 \leqq p \leqq 17)$, $3 \cdot 5 \cdot 7 \cdot 11 p(13 \leqq p \leqq 41), 3 \cdot 5 \cdot 7 \cdot 13 p(17 \leqq p \leqq 41), 3 \cdot 5 \cdot 7 \cdot 17 \cdot 19,3 \cdot 5 \cdot 11 \cdot 13 \cdot 17 \cdot 19$, $3 \cdot 5^{2} 11 \cdot 13 p(17 \leqq p \leqq 31), 3 \cdot 5^{2} 11 \cdot 17 \cdot 19,3 \cdot 5^{3} 7 \cdot 19,3^{2} 7 \cdot 11 \cdot 13 p(17 \leqq p \leqq 31)$, $3^{2} 7 \cdot 11 \cdot 17 \cdot 19,3^{2} 5 \cdot 11 \cdot 13,3^{2} 5 \cdot 11 \cdot 17,3^{2} 5 \cdot 13 \cdot 17 \cdot 19,3^{2} 5^{2} 11 \cdot 19,3^{2} 5^{2} 13 p(17 \leqq p \leqq 23)$, $3^{3} 7 \cdot 11 \cdot 13 p(17 \leqq p \leqq 31), 3^{2} 5^{3} 17 \cdot 19,3^{3} 7 \cdot 13 \cdot 17 \cdot 19,3^{3} 5 \cdot 11 \cdot 19,3^{3} 5 \cdot 13 \cdot 17$, $3^{3} 5 \cdot 13 \cdot 19,3^{3} 5^{2} 11,3^{3} 5^{2} 17 \cdot 19,3^{4} 7 \cdot 11 \cdot 13,3^{4} 5 \cdot 13$.

This improvement gives us

$$
0.2441<A(2)<0.2909 \text {, }
$$

a slight improvement over the bounds 0.241 and 0.314 given by Behrend [1].

We remark that if $k$ is replaced by a proper squarefree multiple of itself, the $M$ of (4) decreases toward zero two ways: the fraction $\varphi(k) / k$ decreases, and the product decreases toward $6 / \pi^{2}$. Here, then, is a way to improve bounds. However, increasing by one the number of primes which divide $k$ at least doubles the number of summands in any truncated version of (1). To obtain our bounds in a reasonable amount of time, we were forced to keep relatively small the number of terms in our summation. This is why our bounds for $A(2)$ are only slightly better than those given by Behrend.

However, our purpose was to compute bounds at enough points to obtain some 
TABLE I

\begin{tabular}{cccccc}
\hline$x$ & $\begin{array}{l}\text { lower } \\
\text { bound }\end{array}$ & $\begin{array}{l}\text { upper } \\
\text { bound }\end{array}$ & $x$ & $\begin{array}{l}\text { lower } \\
\text { bound }\end{array}$ & $\begin{array}{l}\text { upper } \\
\text { bound }\end{array}$ \\
\hline 1.0 & 1.000 & 1.000 & 3.0 & .0186 & .0461 \\
1.1 & .7852 & .8305 & 3.1 & .0131 & .0384 \\
1.2 & .7434 & .7684 & 3.2 & .0084 & .0322 \\
1.3 & .6855 & .7134 & 3.3 & .0058 & .0276 \\
1.4 & .6149 & .6495 & 3.4 & .0040 & .0239 \\
1.5 & .5678 & .6045 & 3.5 & .0025 & .0213 \\
1.6 & .4515 & .5024 & 3.6 & .0014 & .0192 \\
1.7 & .4096 & .4507 & 3.7 & .0009 & .0176 \\
1.8 & .3437 & .3909 & 3.8 & .0005 & .0164 \\
1.9 & .2813 & .3359 & 3.9 & .0003 & .0154 \\
2.0 & .2441 & .2909 & 4.0 & .0001 & .0147 \\
2.1 & .1918 & .2372 & 4.1 & .0000 & .0141 \\
2.2 & .1548 & .2022 & 4.2 & .0000 & .0137 \\
2.3 & .1304 & .1741 & 4.3 & .0000 & .0134 \\
2.4 & .1032 & .1441 & 4.4 & .0000 & .0131 \\
2.5 & .0846 & .1225 & 4.5 & .0000 & .0129 \\
2.6 & .0625 & .0990 & 4.6 & .0000 & .0127 \\
2.7 & .0465 & .0821 & 4.7 & .0000 & .0125 \\
2.8 & .0354 & .0671 & 4.8 & .0000 & .0124 \\
2.9 & .0242 & .0552 & 4.9 & .0000 & .0123 \\
& & & 5.0 & .0000 & .0122 \\
\hline
\end{tabular}

idea of the behavior of $A(x)$, and not merely to refine the bounds given by Behrend, for the points at which Behrend computed bounds were, as explained below, atypical.

It follows from (8) that each of the summands $A(x, j, k)$ of (1) is essentially the same as $A(x, 1, k)$, dilated horizontally and contracted vertically to a maximum height of $\varphi(k) / j k$. It then follows that the sharp drop (the derivative is known not to exist) which occurs at $x=1$ will also occur at any value $x$ for which there is an integer $n$ such that $x=\sigma(n) / n$. Behrend presented bounds for $A(x)$ at $x=3 / 2,4 / 3,6 / 5$, and 2, corresponding to $n=2,3,5$, and 6, respectively; to the right of each of these points, both our upper and our lower bounds drop sharply: for example, our upper bound at $x=1.51$ is less than our lower bound at $x=1.50$. In view of the explanation above, we were not surprised to also observe sharp drops in our bounds at $x=7 / 4=$ $\sigma(4) / 4$, at $x=9 / 5=\sigma(10) / 10$ and at $x=8 / 7=\sigma(7) / 7$, as well as at several other values of $x=\sigma(n) / n$ with $n$ small.

Mathematics Department

East Texas State University

Commerce, Texas 75428

Mathematics Department

Texas A \& M University

College Station, Texas 77843 
Mathematics Department

Texas A \& M University

College Station, Texas 77843

1. F. Behrend, “Ưber 'numeri abundantes.' II,” Preuss. Akad. Wiss. Sitzungsber., v. 6, 1933, pp. 280-293.

2. H. Davenport, "Úber 'numeri abundantes,'" Preuss. Akad. Wiss. Sitzungsber., v. 26/29, 1933 , pp. 830-837.

3. C. WALL, Topics Related to the Sum of Unitary Divisors of an Integer, Ph.D. Dissertation, University of Tennessee, Knoxville, Tenn., 1970. 